\title{
On Post-Resonance Backward Whirl in an Overhung Rotor with Snubbing Contact
}

\author{
Mohammad A. AL-Shudeifat ${ }^{1}$, Michael Friswell ${ }^{2}$, Oleg Shiryavev ${ }^{3}$, C. Nataraj ${ }^{4}$ \\ ${ }^{1}$ Department of Aerospace Engineering, Khalifa University of Science and Technology, \\ Abu Dhabi, PO Box 127788, United Arab Emirates, mohd.shudeifat@ku.ac.ae \\ ${ }^{2}$ College of Engineering, Swansea University, Bay Campus \\ Fabian Way, Crymlyn Burrows Swansea SA1 8EN, UK, m.i.friswell@swansea.ac.uk \\ ${ }^{3}$ Department of Mechanical Engineering, University of Alaska Anchorage, 3211 \\ Providence Dr., Anchorage, AK 99508, USA, oshiryayev@alaska.edu \\ ${ }^{4}$ Villanova Center for Analytics of Dynamic Systems, Villanova University, 800 Lancaster \\ Ave., Villanova, PA 19085, USA, c.nataraj@villanova.edu
}

\begin{abstract}
Rotordynamic systems are central to many aerospace and heavy-duty industrial applications. The vibrational response of such systems is usually associated with forward whirl (FW) and backward whirl (BW) precessions. It is well-known in the literature that the BW precession generally precedes the passage through the critical FW resonance precession. Therefore, it can be named as a pre-resonance BW frequency ( $\mathrm{Pr}-\mathrm{BW})$. However, another kind of BW has been recently observed to be immediately excited after the passage through the critical FW resonance frequency in cracked rotors with anisotropic supports during run up and coast down operations. Consequently, this kind of BW can be named as a post-resonance backward whirl (Po-BW) precession. The Pr-BW and Po-BW phenomena are investigated here with an overhung rotor system that exhibits snubbing contact and stiffness anisotropy in the supports. Incorporating the snubbing moment couple into the equations of motion of the considered overhung rotor model yields a piecewise and strongly nonlinear system. Full spectrum analysis (FSA) is employed to capture the BW zones of rotational speeds in the whirl response. Wavelet transform spectrum analysis is also employed to determine the frequency content in the Pr-BW and the Po-BW zones. Three cases are considered in this numerical study to explore the effect of the support stiffness isotropy and anisotropy with active and inactive snubbing contact on the Po-BW excitation. For all cases, the Po-BW zones of rotational speeds are found. Moreover, the broadness and recurrence of the Po-BW zones of rotational speeds are more prominent for the cases of active snubbing contact. Even though the Pr-BW and Po-BW zones are excited at different shaft rotational speeds, they are found to possess nearly similar BW frequencies which are less than the FW resonance frequency of the considered system.
\end{abstract}

Keywords: Backward whirl, overhung rotor, post-resonance backward whirl, full spectrum analysis, rotor system

\section{Introduction}

It is important to understand the dynamic behavior of rotor systems during their start-up and run-down because large vibration amplitudes that may occur during these operations may cause excessive stresses, and lead to accumulation of fatigue 
damage, reducing in-service lifetime. In addition to large stresses in the rotor shaft, excessive vibration amplitudes may cause rubbing of rotor parts against the stator, which could cause destructive effects on bearings, seals, and other parts [14].

Several previous studies on the behavior of accelerated rotors have focused on determining the maximum amplitude of whirling, which is related to the stresses experience by the machine. Howitt [5] investigated the effect of acceleration on the critical speeds and the maximum amplitude of whirl for simply supported vertically oriented slender rotors. For an accelerating rotor, the critical speed will increase, while the amplitude would decrease proportionally to the square root of angular acceleration. Zhou and Shi [6] derived an analytical approach for computing the transient vibration response of a Jeffcott rotor undergoing constant acceleration. Later they proposed an adaptive active balancing strategy for suppression of vibration in accelerating rotors [7]. There were additional studies on accelerated rotors whose main aim was to investigate methods for reducing the maximum amplitude of lateral response during acceleration [8-10].

One particularly worrying effect that is usually associated with large lateral motions and rubbing is backward whirl (BW). Bartha [11] presented a thorough study on excitation of BW during steady state operation. Rubbing contact may also occur in situations when bearing clearances are small and rotor shafts are relatively light and flexible. In such situations, the gravitational force will have a significant effect on the dynamics of the rotor.

We now present an overview of previous work focused on the dynamics of overhung rotors. Qin et al. [12] studied the response of an overhung rotor with rubbing impact in steady state operating regimes. They found that the system is 
able to exhibit a variety of nonsynchronous motions such as subharmonics, quasiperiodic motions, and chaotic responses, which are affected by the magnitude of unbalance excitation, the characteristics of the supports, and the amount of external damping. Similar results were observed in an earlier study of a simply supported multi-disc rotor with rubbing [13].

Abdul Azeez and Vakakis [14] studied the behavior of an overhung rotor undergoing rotor-to-stator impacts using a distributed parameter model that was discretized by employing the assumed modes method. The model included gyroscopic effects and has been used to investigate quasi-steady state operating regimes. They were able to observe subsynchronous response components in both numerically generated and experimental data.

Ma et al. [15] studied the dynamics of an overhung rotor with parallel and angular misalignments and the effect of oil film instability during start-up and coast-down operations. The authors noted the rich spectral composition of the rotor response, and that the amplitudes of various harmonics present in the response are more obvious under parallel misalignment than under angular misalignment conditions. Another study on the prediction of self-excited instabilities involving rotor rub was performed by Fan et al. [16].

Fu et al. $[17,18]$ developed a method for efficient computation of dynamic response amplitudes for steady state operating regimes of overhung rotors with uncertain parameters. They showed that relatively small uncertainties in system parameters can have significant effects on the dynamic behavior of rotors. This work was later extended to include the effects of rubbing [19]. 
$\mathrm{Fu}$ et al. [20] studied the transient response of an accelerating overhung hollow rotor where the Polynomial Chaos Expansion and the Chebyshev Surrogate Method were used in the analysis with uncertain physical parameters. Numerical simulations were performed for a wide range of uncertainties in the system physical parameters within bounded intervals. The obtained whirl response shows several zones of local transient peaks that immediately appear after passing through the resonance rotational speed. Therefore, these zones are suggestive for Po-BW motion if the stiffness symmetry is altered. Chipato et al. [21,22] studied the dynamics of an overhung rotor with frictional contact between the rotor and the stator. The first investigation focused on the effect of gravity on the dynamic response of flexible rotors [21]. In the following work [22], they investigated the behavior of rotors systems with stiff shafts, where gravity can be neglected. The response of the system was characterized for different values of friction coefficient and unbalance excitation magnitudes. This work illustrated the existence of several different types of bouncing motion that this system may exhibit depending on the presence of friction, including partial annular and full annular contact. It is observed that increasing the unbalance excitation force and the coefficient of friction increase the response region with BW.

Another study on the dynamics of an overhung rotor was recently presented by Bisoi et al. [23]. They considered a rotor with a motor that can supply a limited amount of power, i.e. a non-ideal drive, and studied the influence of various parameters on the appearance of the Sommerfeld effect in the system. They showed that under conditions when rotary inertia is high and the drive motor power is limited, the rotor system may have difficulty in passing through the 
critical speed, because much of the supplied energy will be transferred to the lateral vibration of the rotor instead of increasing its rotational speed.

Wu et al. [24] utilized a 4-DOF model for an overhung rotor with anisotropic supports to represent the experimental rig, which they used to validate their inhouse software for data processing and plotting full-spectrum cascade plots against those produced with commercial software. They were able to observe BW orbits that arise when passing through resonance, but did not investigate this phenomenon further as the focus of their work was on validation of their data processing software.

In steady state regimes, critical forward whirl (FW) and BW speeds can be predicted by the Campbell diagram. From the literature, it is well known that in this case the $\mathrm{BW}$ rotational speed precedes the $\mathrm{FW}$, and we refer to this phenomenon as the pre-resonance BW (Pr-BW). Recently, another type of BW was discovered [25]. It occurs after the passage through the FW critical speed, so we refer to it as the post-resonance BW (Po-BW). Its appearance is affected by several factors such as the degree of anisotropy of bearings, angular acceleration rate, and others. This phenomenon has not yet been thoroughly investigated in rotor systems with overhung disks.

In this work, we present a study of an overhung rotor system that exhibits snubbing contact and stiffness anisotropy in the supports. Incorporating the snubbing moment couple into the equations of motion of the considered overhung rotor model, yields a piecewise and strongly nonlinear system. In this study, the capability of the full spectrum analysis in localizing the BW motion is utilized. In addition, the wavelet transform spectrum analysis is employed to determine the time dependent frequency content in the Pr-BW and the Po-BW zones. Three 
cases are considered in this numerical study to explore the effect of the support stiffness isotropy and anisotropy with the active and inactive snubbing contact on the Po-BW excitation. In the following sections we present the details of the numerical models and the results obtained in each case.

\section{Theoretical Model}

The two-degrees-of-freedom lumped overhung rotor model derived in [26] and later employed in $[21,22]$ is considered here for studying the Pr-BW and Po-BW phenomena in the system with snubbing frictional contact. This model only considers angular oscillations which allows the gyroscopic effect to be incorporated into the equations of motion. In addition, solving the equations of motion for the angular oscillations enables the calculation of the transverse horizontal and vertical oscillations which makes this model more practical than the classical Jeffcott rotor model. The schematic diagram representing this model is shown in Fig. 1. The model represents a stiff rotor with flexible supports where the rigid disk is attached to the supports at the origin of the coordinates system by a stiff massless shaft as shown. The mass center of the disk is allowed to oscillate by angles $\theta$ and $\psi$ about the $x$ and $y$ axes, respectively. The angular restoring stiffness coefficients in both angular oscillation directions are represented by $k_{\theta}$ and $k_{\psi}$, respectively. Similarly, the angular damping coefficients are represented by $C_{\theta}$ and $C_{\psi}$, respectively. 


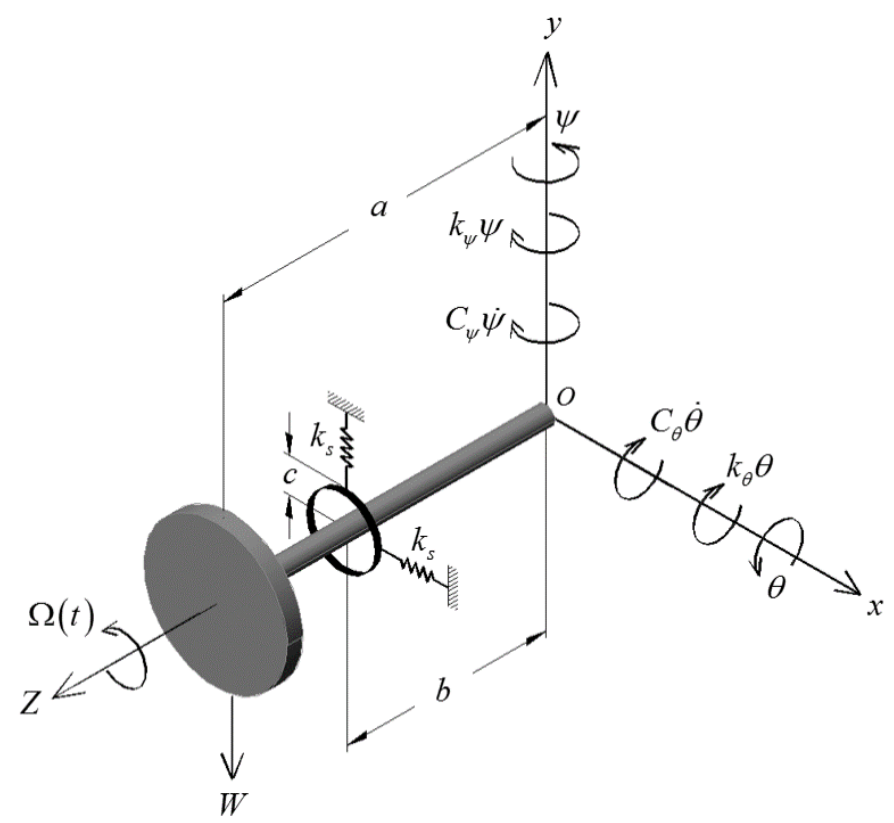

Fig. 1 Schematic diagram of the considered overhung rotor model.

The model also incorporates horizontal and vertical restoring snubbing stiffness which is represented by $k_{s}$ in both $x$ and $y$ axes directions. The contact occurs when the radial displacement of the mass center of the disk satisfies $r_{c}=c^{*}$ where $c^{*}=c a / b$. Therefore, during the instant of contact, a snubbing restoring moment couple is generated which is associated with the normal snubbing forces.

Accordingly, the system exhibits a piecewise nonlinear dynamical behavior. The resulting snubbing moment couple includes the components $M_{\theta}$ about the $x$-axis and $M_{\psi}$ about the $y$-axis. In addition, considering ideal Coulomb friction during the contact as in with friction coefficient $\mu$, the resulting friction moments about the $x$ and $y$ axes, $M_{\theta}$ and $M_{\psi}$, are given according to [3] as 


$$
\begin{array}{ll}
M_{\theta}=k_{s} b^{2}(\theta-\mu \psi)\left(1-\frac{c}{b \sqrt{\theta^{2}+\psi^{2}}}\right), & \left|\mathbf{r}_{c}\right| \geq c^{*} \\
M_{\psi}=k_{s} b^{2}(\psi+\mu \theta)\left(\frac{c}{b \sqrt{\theta^{2}+\psi^{2}}}-1\right), & \left|\mathbf{r}_{c}\right| \geq c^{*}
\end{array}
$$

where $M_{\theta}=M_{\psi}=0$ for $\left|\mathrm{r}_{c}\right|<c^{*}$.

The rotor is assumed to have a constant angular acceleration rate, $\alpha$, about the $Z$ axis, and hence the rotation angle is $\beta=0.5 \alpha t^{2}(\mathrm{rad})$ and the angular velocity is $\Omega(t)=\alpha t(\mathrm{rad} / \mathrm{s})$, assuming zero initial angular displacement and velocity.

Therefore, the derivation of the linear-time-varying (LTV) governing equations of motion, given in the appendix, incorporates the acceleration rate, unbalance force, weight, snubbing force moment couple and the friction effect. Accordingly, these equations are written as

$$
\begin{aligned}
& I_{0} \ddot{\theta}+I_{p} \alpha t \dot{\psi}+k_{\theta} \theta+C_{\theta} \dot{\theta}=m g a-m e a\left((\alpha t)^{2} \sin \left(0.5 \alpha t^{2}\right)-\alpha \cos \left(0.5 \alpha t^{2}\right)\right)+M_{\theta} \\
& I_{0} \ddot{\psi}-I_{p} \alpha t \dot{\theta}+k_{\psi} \psi+C_{\psi} \dot{\psi}=m e a\left((\alpha t)^{2} \cos \left(0.5 \alpha t^{2}\right)+\alpha \sin \left(0.5 \alpha t^{2}\right)\right)+M_{\psi}
\end{aligned}
$$

where $m$ represents the mass of the rigid disk, $e$ is the mass unbalance eccentricity of the disk, $I_{0}$ is the diametral moment of inertia of the shaft-disk assembly and $I_{p}$ is the polar moment of inertia of the rigid disk. $I_{0}$ and $I_{p}$ are given by

$$
I_{0}=m\left(\frac{D^{2}}{16}+\frac{h^{2}}{12}+\left(a+\frac{h}{2}\right)^{2}\right), \quad I_{p}=m \frac{D^{2}}{8}
$$

where $D$ and $h$ are the diameter and thickness of the disk. The physical parameters in the numerical simulation analysis, assuming a rigid steel disk-shaft rotor assembly, are disk width $h=0.05 \mathrm{~m}$, disk diameter $D=0.3 \mathrm{~m}$, disk density 
$\rho_{d}=7810 \mathrm{~kg} / \mathrm{m}^{3}$, shaft length $a=0.5 \mathrm{~m}$, snubbing force location $b=0.35 \mathrm{~m}$,

radial clearance $c=1.8621 \times 10^{-4} \mathrm{~m}$, angular damping coefficients

$C_{\theta}=C_{\psi}=30 \mathrm{~N} \cdot \mathrm{m} \cdot \mathrm{s} / \mathrm{rad}$, angular stiffness coefficients at the support of

$k_{\theta}=10^{6} \mathrm{~N} \cdot \mathrm{m} / \mathrm{rad}$ and $k_{\psi}=\sigma k_{\theta}$ where $\sigma$ represents the supports stiffness ratio,

and snubbing force stiffness $k_{s}=\rho k_{\theta}$ where $\rho$ is the stiffness ratio. The product

of the disk mass and the eccentricity, that is the mass unbalance magnitude in Eq.

(2), is selected as $m e=10^{-4} \mathrm{~kg} \cdot \mathrm{m}$ for all numerical simulations. In addition, the

horizontal and vertical displacements of the disk mass center in $x$ and $y$ directions, respectively, are extracted from the numerical simulation response of $\theta$ and $\psi$ as

$$
\begin{aligned}
& u=a \psi \\
& v=-a \theta
\end{aligned}
$$

Accordingly, the radial displacement vector of the disk mass center is calculated by $\mathbf{r}_{c}=u \hat{\mathbf{i}}+v \hat{\mathbf{j}}$ where its magnitude is calculated from $r_{c}=\left|\mathbf{r}_{c}\right|=\sqrt{u^{2}+v^{2}}$. To determine the precision direction of the whirl orbit, at consecutive time instants of $t^{(i)}$ and $t^{(i+1)}$, the position vectors of the rigid disk mass center are represented by $\mathbf{r}_{c}^{(i)}=u^{(i)} \hat{\mathbf{i}}+v^{(i)} \hat{\mathbf{j}}$ and $\mathbf{r}_{c}^{(i+1)}=u^{(i+1)} \hat{\mathbf{i}}+v^{(i+1)} \hat{\mathbf{j}}$, respectively. Therefore, the shaft is in FW precession if the resultant vector of $\mathbf{r}_{c}^{(i)} \times \mathbf{r}_{c}^{(i+1)}$ is in the positive $z$-axis direction, otherwise, it is in BW precession.

The accuracy of the numerical simulation response requires identification of the precise time of contact between the shaft and the snubbing stiffness zone which is numerically calculated at the instant of contact with $10^{-10}$ precision using the Runge-Kutta fourth order method. In addition, convergence in the numerical simulation response required the time step for the numerical simulation to be less 
than $2 \times 10^{-5} \mathrm{~s}$. Otherwise, using the Heaviside function or insufficient numerical integration time steps do not guarantee the accuracy for such a strongly nonlinear piecewise dynamical system.

Full-spectrum-analysis (FSA) [27,28] is employed with the numerical simulation response to identify the zones of BW rotational speed and the wavelet transform spectrum [29] is also applied to identify the frequency content in these zones. The position of the rotor centerline is determined using two orthogonal coordinates. Application of conventional FFT to the data measured along each of the coordinate directions results in half-spectrums that do not allow determination of rotor precession (FW or BW). In FSA, the orbit of the rotor at each frequency value is represented as a summation of two counter-rotating vectors: one is rotating forward (same direction as the rotor rotation) and the other is rotating backward (opposite to the direction of rotor rotation). If the rotor undergoes FW, then the magnitude of the forward rotating vector is larger than that of the backward rotating vector. Alternatively, the rotor undergoes BW when the magnitude of the backward rotating vector is larger than that of the forward rotating vector. Hence, the FSA allows one to determine the direction of rotor precession, which has been widely utilized in condition monitoring of rotating machinery. In addition, the wavelet transform analysis is useful for determining the frequency content of transient signals as they evolve with time, which makes it particularly suitable for the analysis of an accelerating rotor.

\section{Snubbing-Free System with Anisotropic Stiffness}

Here, the snubbing moment couple is not incorporated within the model to distinguish between the pre-resonance $\mathrm{BW}(\mathrm{Pr}-\mathrm{BW})$ and the post resonance $\mathrm{BW}$ (Po-BW) based on the stiffness anisotropy in the support. Therefore, unequal 
stiffness coefficients are employed at the support for the snubbing-free model. Accordingly, the effect of the stiffness ratio $\sigma=k_{\psi} / k_{\theta}$ at the support on the FW and BW is first investigated using the critical speeds predicted by the Campbell diagram at each value of $\sigma$, which are plotted in Fig. 2. It is well-known that the Pr-BW is usually associated with the Campbell diagram prediction at constant angular velocities (steady-state operations). However, it has been recently found [6] that the Po-BW is associated with transient run up and coast down operations during which a passage through the critical forward resonance frequency takes place.

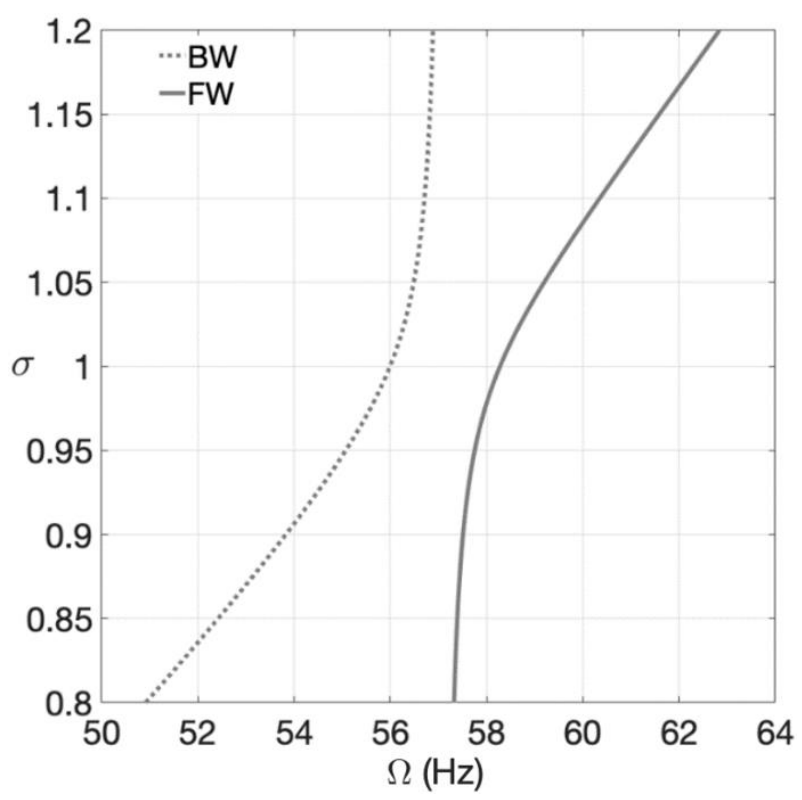

Fig. 2 Critical speeds predicted by the Campbell diagram for the FW and BW resonance frequencies versus $\sigma$.

Fig.3 shows the zones of the Pr-BW and Po-BW (black regions) of the numerical whirl response, the critical BW and FW frequencies obtained from the numerical whirl response (solid and dotted blue lines, respectively), and the BW and FW curves obtained by the Campbell diagram results (dotted and solid gray lines, respectively). At a very low angular acceleration rate in Fig. 3a, only the Pr-BW zones are excited for different values of $\sigma$. In addition, the critical FW and BW 
frequencies obtained at the peak values of the numerical whirl response are slightly shifted to higher values compared with the Campbell diagram results. However, for higher values of the angular acceleration rates in Figs. 3b-3d the shift in the FW resonance frequency is further increased. Zones of the Po-BW start to appear at higher acceleration rates as shown in Figs. 3b-3d where their recurrence and extent are significantly affected by the acceleration rate. In addition, it is also observed that the Pr-BW zones start to shrink and split into multi zones as the acceleration rate increases.
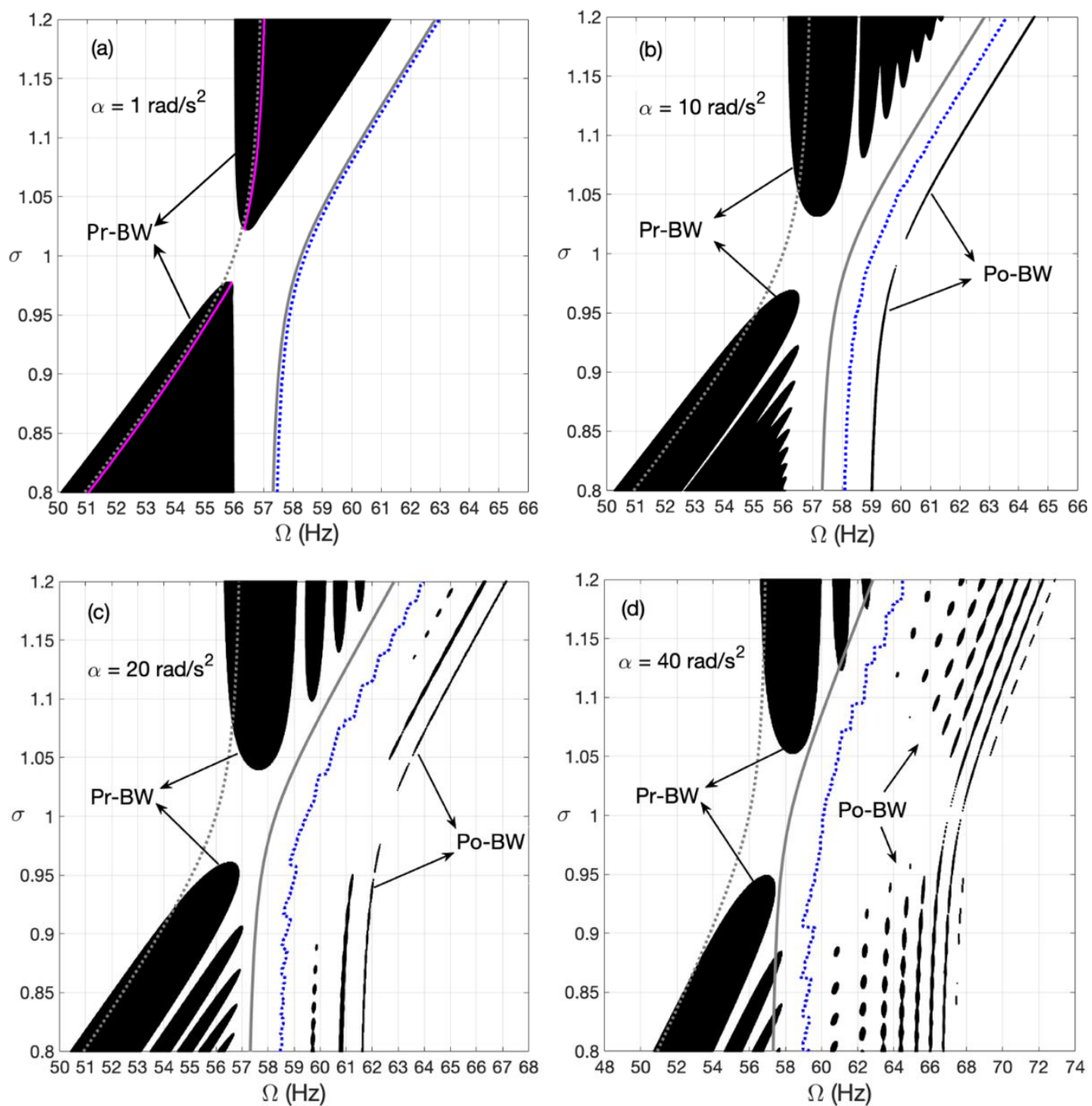

Fig. 3 The Pr-BW and Po-BW zones in (a) - (d) for various values of $\alpha$ and $\sigma$ where the $\operatorname{Pr}-\mathrm{BW}$ and Po-BW zones are represented by black regions, FW and BW curves of Campbell diagram results are represented by solid and dotted gray lines, respectively, and FW and BW frequencies of the whirl response are represented by dotted and solid blue lines, respectively. 
For $\sigma=0.95$ and $\sigma=0.85$ at $\alpha=20 \mathrm{rad} / \mathrm{s}^{2}$, the whirl response results and their corresponding FSA plots are shown in Fig.4. For the whirl amplitudes response in Figs. $4 \mathrm{a}$ and $4 \mathrm{~b}$, the previously observed zones in Fig. 3 of the Pr-BW and Po-BW are clearly shown. Furthermore, the FSA plots corresponding to the whirl amplitudes responses in Figs. $4 \mathrm{a}$ and $4 \mathrm{~b}$ are shown in Figs. $4 \mathrm{c}$ and $4 \mathrm{~d}$, respectively, which clearly confirms these Pr-BW and Po-BW zones of rotational speeds. In addition, the wavelet transform is applied to the response within the PrBW zones and shown in Figs. 5a and 5b, where the frequency content is similar to that predicted by the Campbell diagram. Since the Po-BW zones are very narrow, the wavelet transform spectrum analysis of these zones does not result in accurate frequency content. However, the wavelet transform spectrum analysis is applied in the following section to accurately capture the frequency content in the Po-BW response for the system with an active snubbing moment couple.
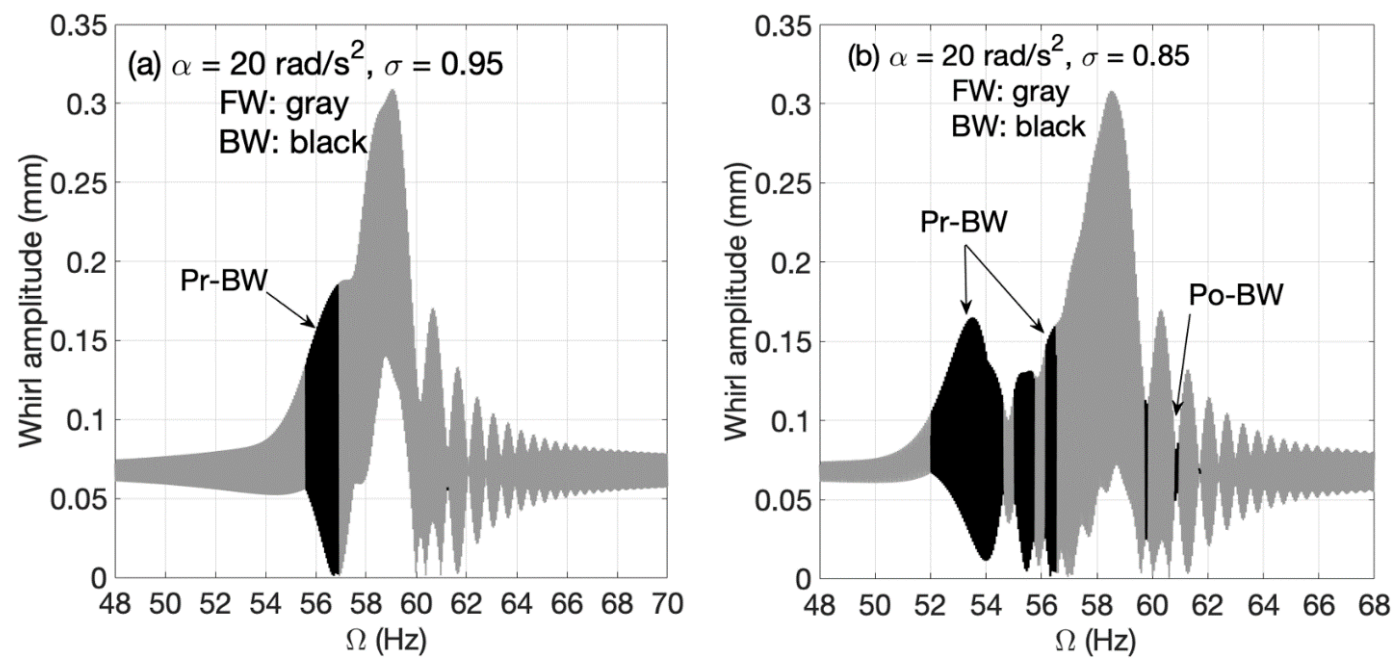

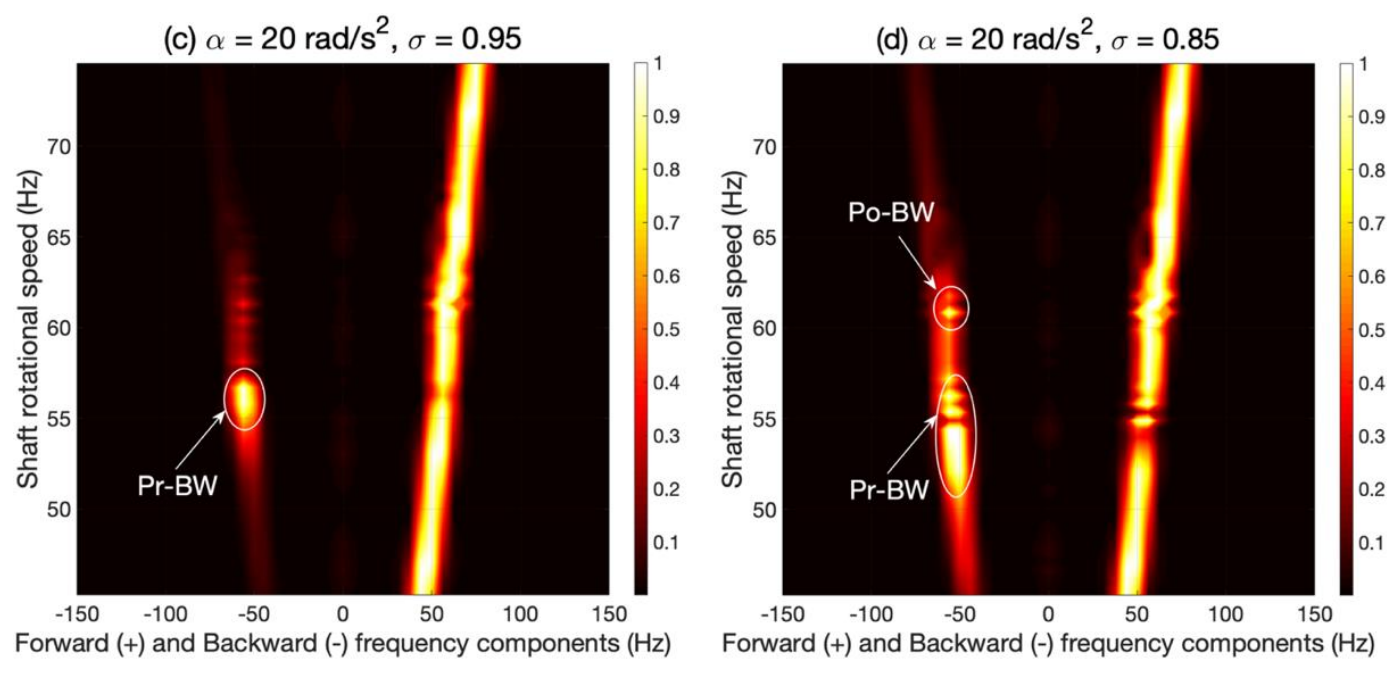

Fig. 4 The resultant whirl amplitudes in (a) and (b), and their corresponding FSA plots in (c) and (d) for selected values of $\alpha$ and $\sigma$.
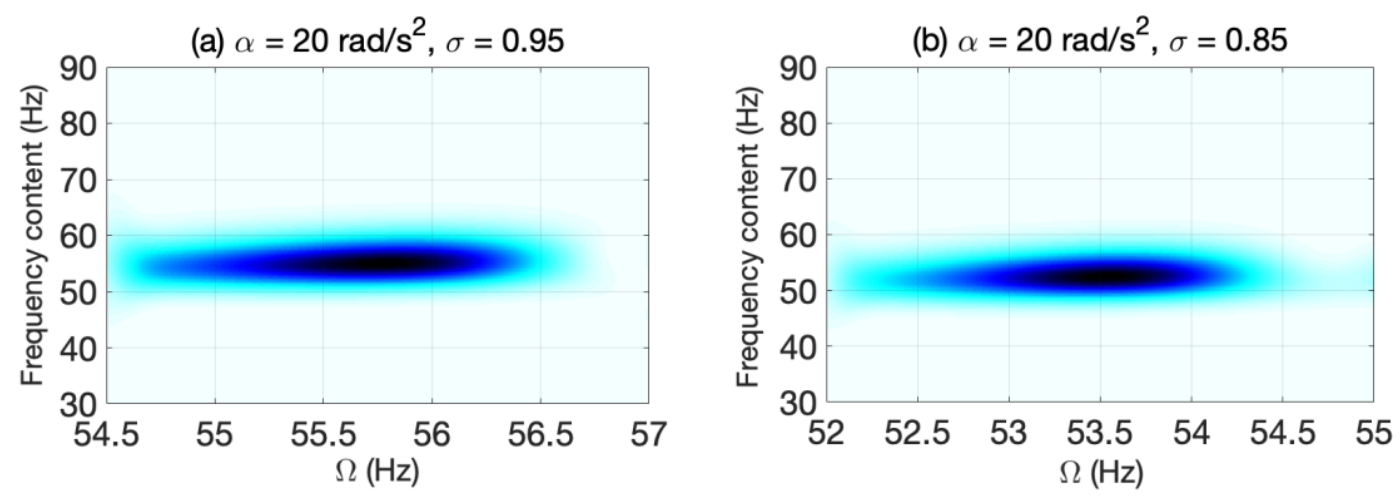

Fig. 5 The wavelet transform frequency content of the Pr-BW zones in Figs. 4a and 4b.

\section{Active Snubbing Force with Isotropic Stiffness}

The snubbing moment couple is now incorporated into the equations of motion for the system with isotropic stiffness content in the support. Therefore, the effect of the ratio of the snubbing stiffness $k_{s}$ to the angular stiffness $k_{\theta}$, which is represented by $\rho=k_{s} / k_{\theta}$ is investigated. First, the effect of the angular acceleration rate on the Po-BW excitation at selected values of $\rho$ is shown in Fig. 6. It is observed that the intensity and recurrence of the Po-BW excitation depends significantly on the value of $\rho$. In addition, it is important to note that for some values of $\alpha$ outside of the selected ranges of the angular acceleration rates in Fig. 6, unstable solutions exist. 

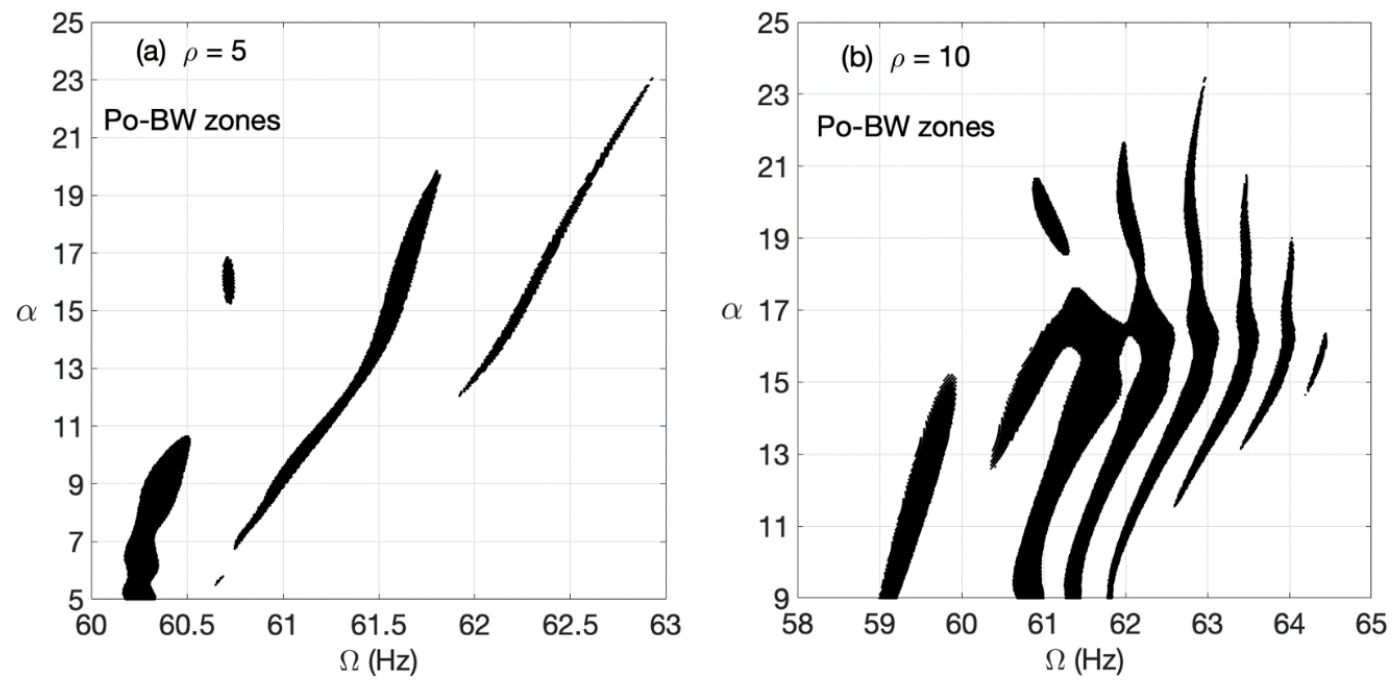

Fig. 6 Effect of the angular acceleration rate $\alpha$ on the Po-BW zones at selected values of $\rho$.

Another investigation is performed by varying values of $\rho$ for the angular acceleration rates $\alpha=10 \mathrm{rad} / \mathrm{s}^{2}$ and $\alpha=20 \mathrm{rad} / \mathrm{s}^{2}$, respectively, where the obtained Po-BW zones of rotational speeds are plotted in Fig. 7. It is observed, for all considered stiffness ratios, that only the Po-BW zones are excited after the passage through the critical FW resonance frequency (dotted blue curve) due to the snubbing moment coupling effect and isotropy in the support stiffness coefficients.

For values of $\alpha=20 \mathrm{rad} / \mathrm{s}^{2}$ and $\rho=15$, the whirl response and its coreesponding FSA plot are shown in Fig.8. In the whirl amplitude response in Figs. 8a, the PoBW zones are clearly excited by the effect of the snubbing moment couple after the passage through the critical FW resonance rotational speed. In addition, these zones are perfectly captured by the corresponding FSA plot shown in Fig. 8b. Moreover, in Fig. 9 the wavelet transform is applied to the named four zones of the Po-BW in Fig. 8a (A, B, C and D). It is interesting that all of these Po-BW zones, which occur at different shaft rotational speeds, have very similar BW 
frequency content that is concentrated at $\omega_{B W} \cong 56 \mathrm{~Hz}$, which is less than the critical FW whirl resonance frequency in the numerical simulation response.
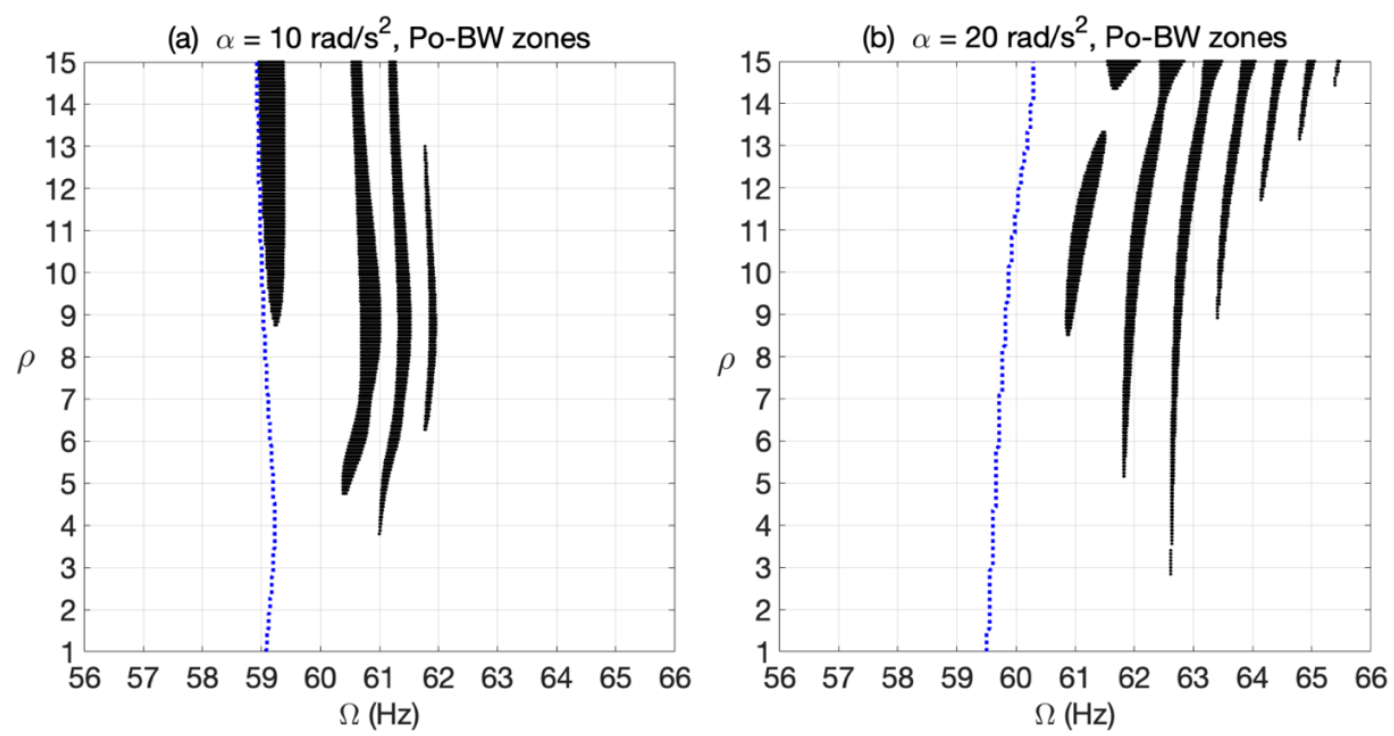

Fig. 7 Effect of the stiffness ratio $\rho$ on the Po-BW zones at selected values of $\alpha$ (dotted blue line for the FW resonance of the numerical whirl response).
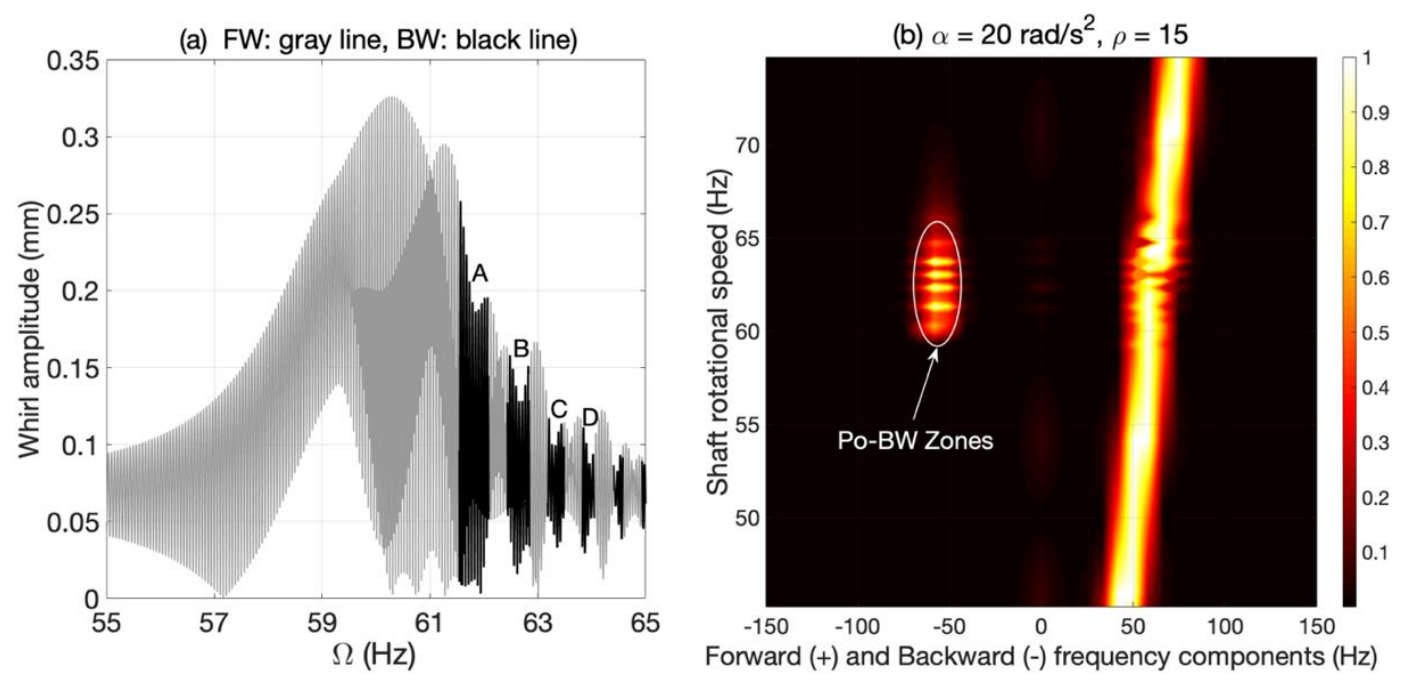

Fig. 8 The resultant whirl amplitude (a), and the corresponding FSA plot (b) for $\alpha=20 \mathrm{rad} / \mathrm{s}^{2}$ and $\rho=15$. 

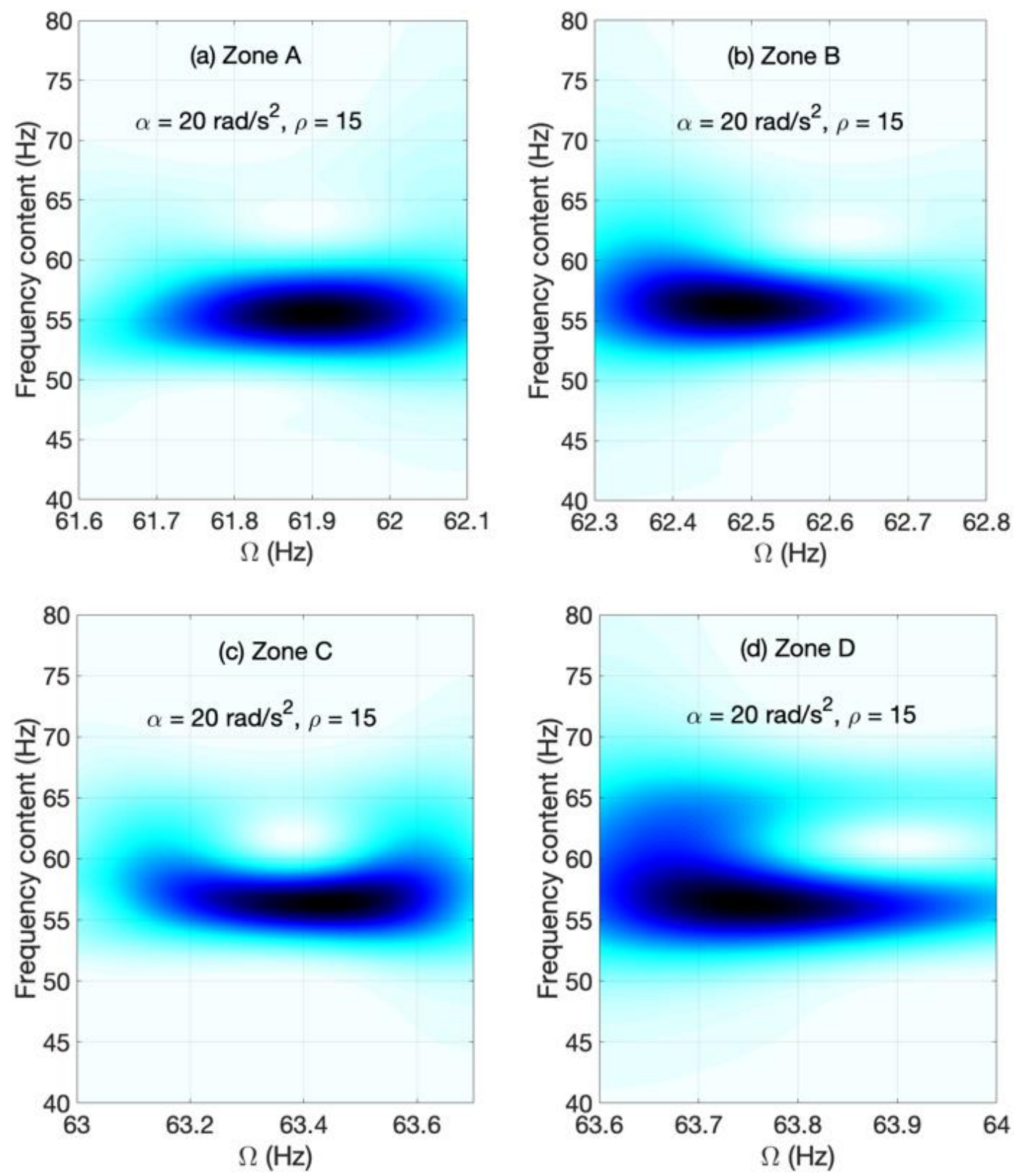

Fig. 9 The wavelet transform frequency spectra of the Po-BW zones in Figs. 8a.

\section{Active Snubbing Force with Anisotropic Support Stiffness}

In the previous sections it has been found that anisotropy in the support excites wider zones of Pr-BW than the Po- BW zones. However, broad and recurrent zones of Po-BW have been excited for the case of active subbing contact with isotropic support. Therefore, the combined effect of an active snubbing contact and anisotropy in the support is investigated here. The results are shown in Figure 
10 for the anisotropic stiffness ratio $\sigma=0.95$ and snubbing stiffness ratios of $\rho=5$ and $\rho=10$ for various angular acceleration rates. It is observed that a stiffer snubbing contact yields more recurrent and intense Po-BW zones at low and intermediate angular acceleration rates than at higher rates. Therefore, it can be stated that the Po-BW response is highly sensitive to the snubbing stiffness ratio and the angular acceleration rate as shown in the figure.
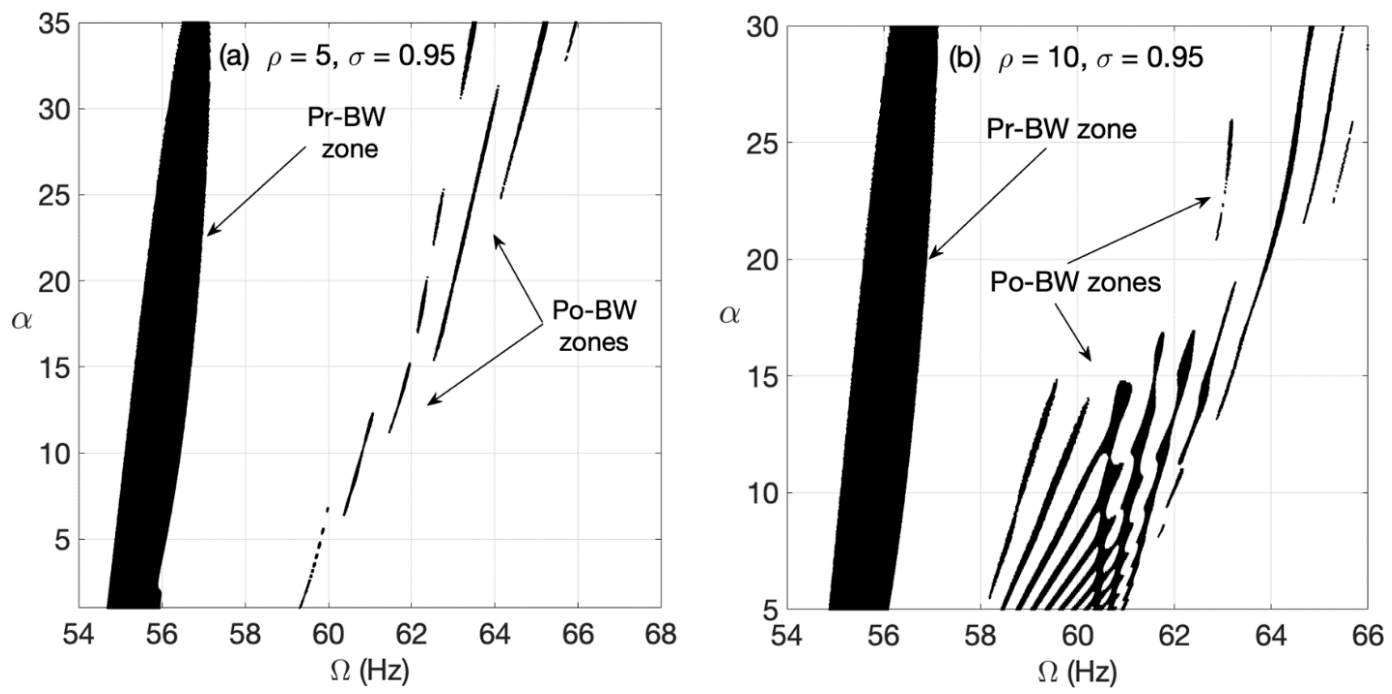

Fig. 10 Effect of stiffness ratios $\rho$ and $\sigma$ on the Pr-BW and Po-BW zones at various values of $\alpha$

For the selected values of $\alpha=10 \mathrm{rad} / \mathrm{s}^{2}, \rho=10$ and $\sigma=0.95$, the whirl response and its corresponding FSA plot are shown in Fig.11. In the whirl amplitude response in Fig. 11a, the Pr-BW and Po-BW zones are both excited before and after the passage through the critical FW resonance rotational speed, respectively, due to the combined effect of the snubbing moment couple and the anisotropic stiffness in the support. In Fig. 11b, these zones are perfectly captured by the corresponding FSA plot. The wavelet transform is applied to the Pr-BW and PoBW zones that are shown in Fig. 11a and the frequency content in these zones is given in Fig. 12. Even though the Pr-BW and Po-BW zones occur at different shaft rotational speeds, they are found to have very similar BW frequency content 
which is concentrated near to $\omega_{B W} \cong 56 \mathrm{~Hz}$. It is important to note that the frequency content in both zones is less than the critical FW whirl resonance frequency in the whirl response.
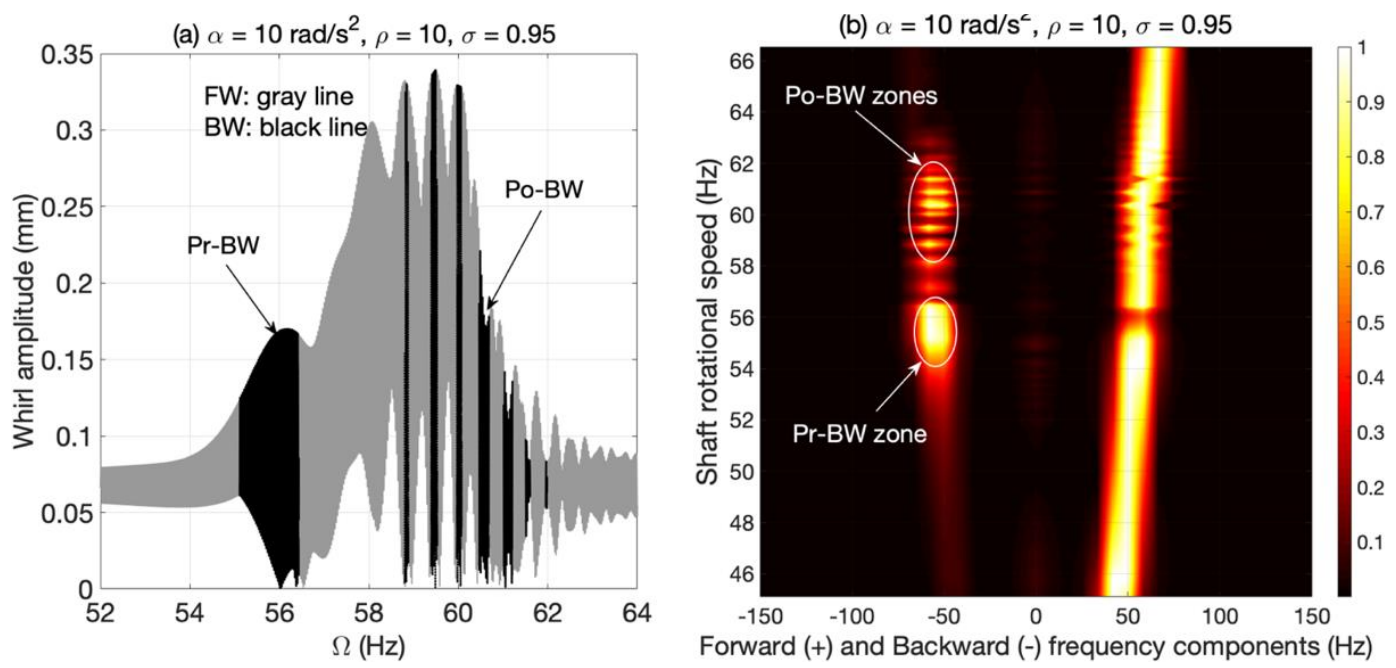

Fig. 11 The resultant whirl amplitude (a), and the corresponding FSA plot (b) for $\alpha=10 \mathrm{rad} / \mathrm{s}^{2}$, $\rho=10$ and $\sigma=0.95$
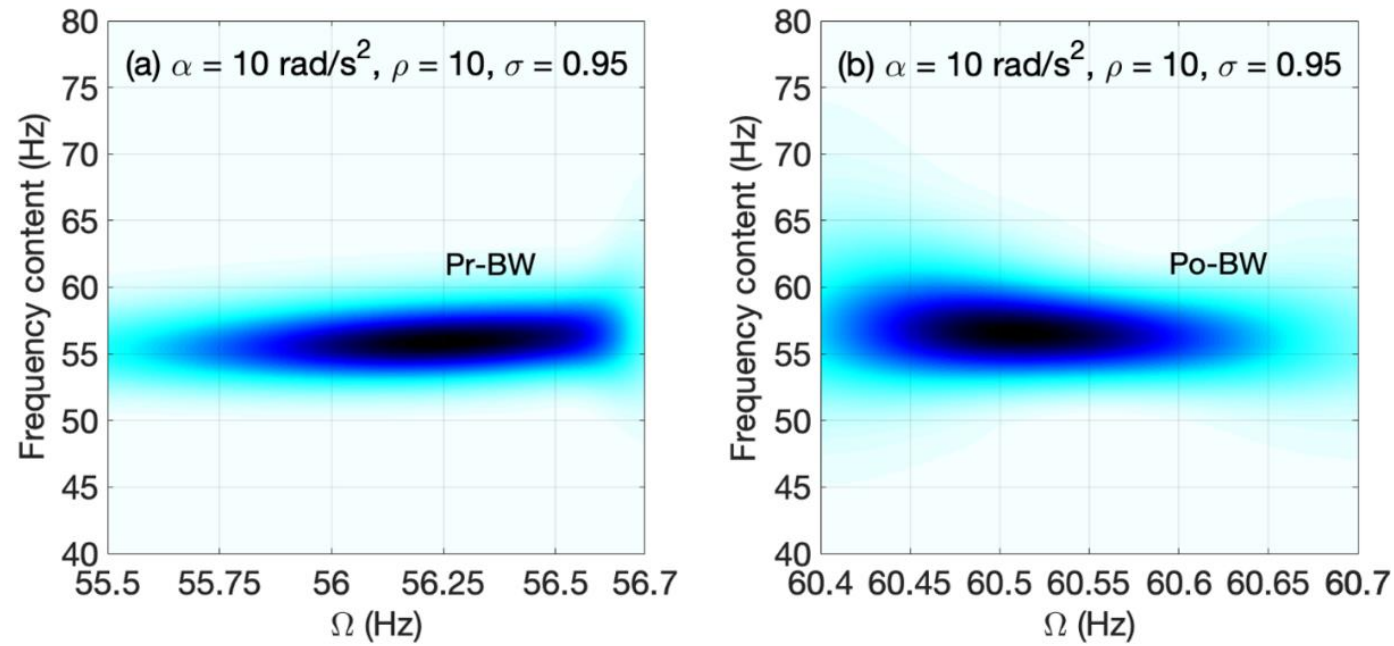

Fig. 12 The wavelet transform frequency spectra of the Pr-BW (a) and Po-BW (b) zones in Fig. 11 .

\section{Rigid Snubbing Boundary}

A nearly rigid snubbing boundary with an extremely high snubbing stiffness ratio is considered here to verify the accuracy, robustness and efficacy of the model and the related numerical analysis. Therefore, the snubbing stiffness ratio is selected 
as $\rho=5 \times 10^{5}$ to provide sufficient rigidity to the snubbing contact boundary. The whirl response results are shown for $\alpha=20 \mathrm{rad} / \mathrm{s}^{2}$ in Fig. 13 for isotropic support stiffness $(\sigma=1)$ and anistropic support stiffness ( $\sigma=0.95)$, respectively. For both cases, the Po-BW zones are clearly excited where the whirl amplitudes in the neigborhood of the resonance FW frequncy are perfectly flattened due to consecutive impacts of the shaft with the nearly rigid snubbing boundary.
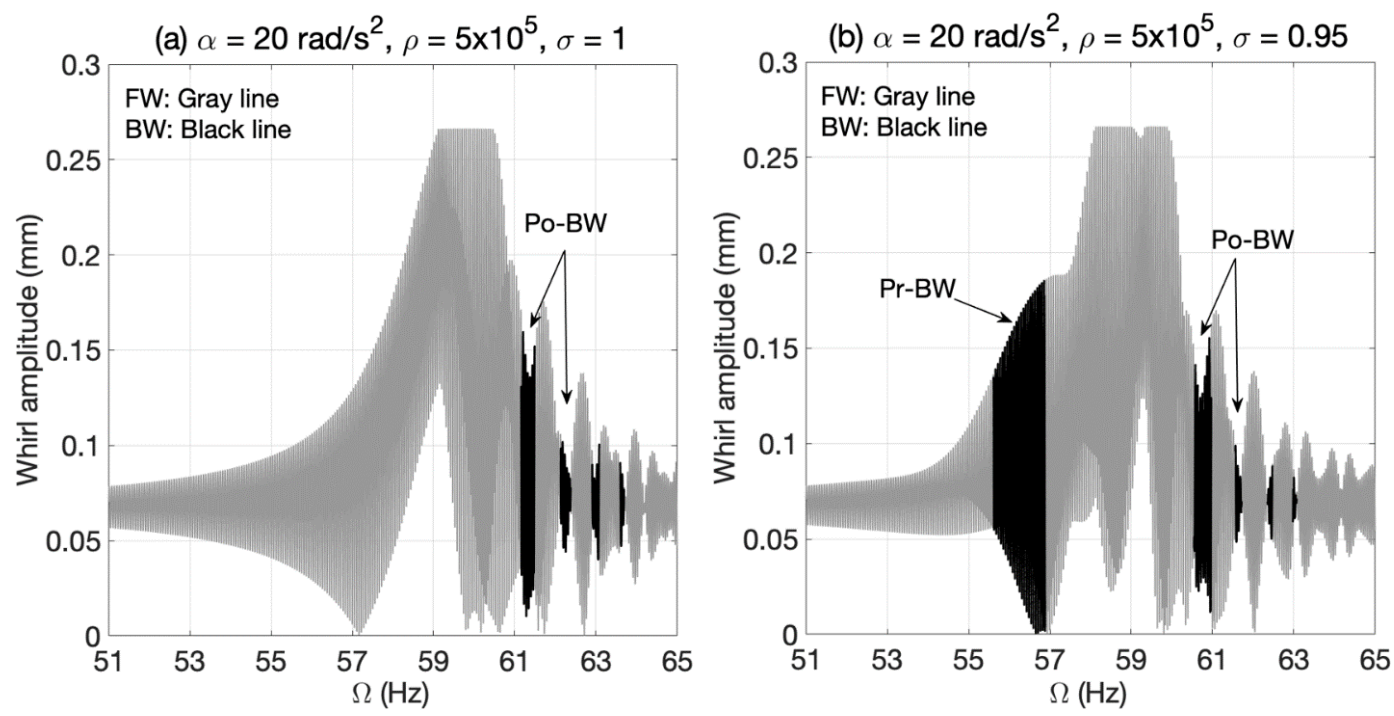

Fig. 13 The resultant whirl amplitudes (a) for isotropic support stiffness, and (b) for an anisotropic support stiffness at very high snubbing stiffness ratio

\section{Supports Cross-Coupling Stiffness}

In this last part of the study, the effect of incorporating a cross-coupling stiffness in the supports $k_{\theta \psi}$ and $k_{\psi \theta}$ on the Po-BW excitation is investigated. The offdiagonal cross-coupling stiffness components in the support stiffness matrix are usually selected to be either zero or significantly lower than the diagonal stiffness components [30-32]. For example, in [31] the off-diagonal cross-coupling stiffness was selected to be equivalent of $1 \%$ of the diagonal stiffness. Accordingly, the results shown in Fig. 14 are obtained based on incorporating the 
cross-coupling stiffnesses $\left(k_{\theta \psi}=k_{\psi \theta}\right)$ in the considered system to be equivalent to $1 \%$ of the previously used diagonal stiffnesses $\left(k_{\theta}=k_{\psi}=10^{6} \mathrm{~N} \cdot \mathrm{m} / \mathrm{rad}\right)$ at $\rho=15$

. For both selected acceleration rates in the figure, the Po-BW zones are still clearly excited. In addition, the comparison between Fig. 8b, in which the crosscoupling stiffness was excluded, and Fig. 14b, in which it is included, highlights that the intensity of the Po-BW is not significantly affected by adding the crosscoupling stiffness to the support stiffness matrix.
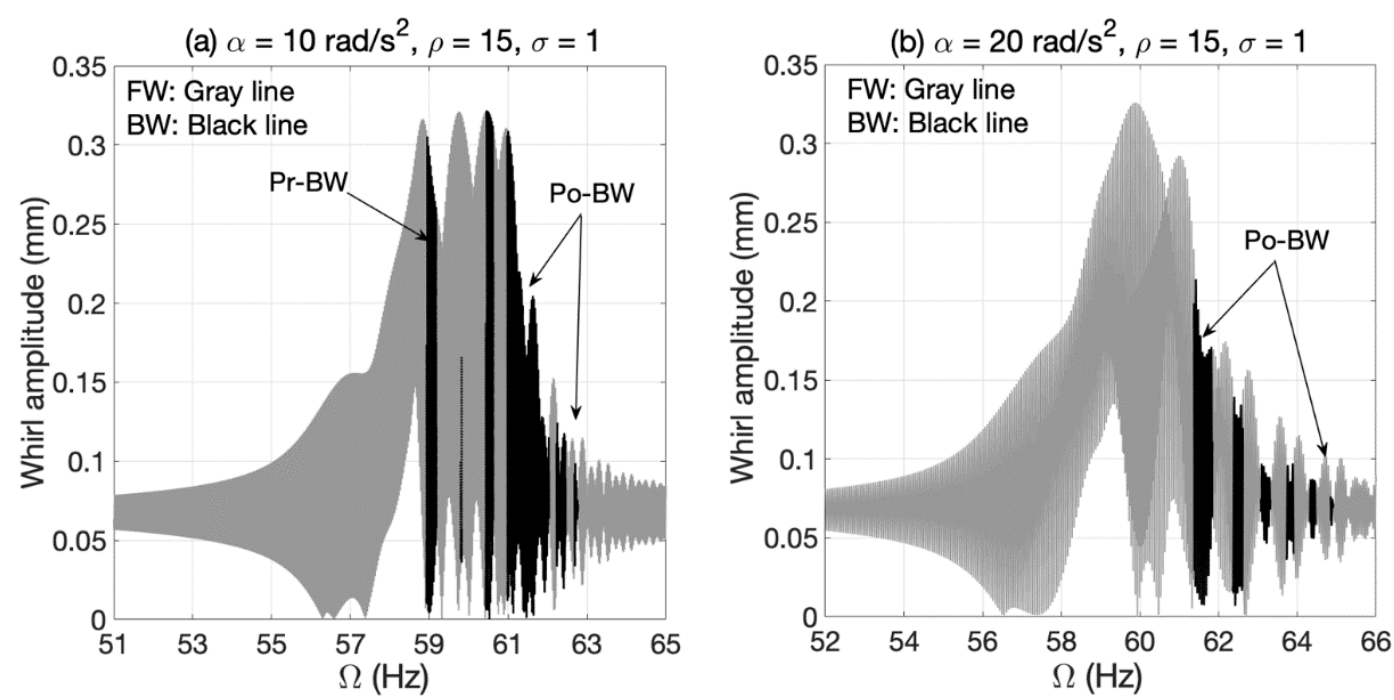

Fig. 14 The resultant whirl amplitudes after incorporating a cross-coupling stiffness in the supports (a) for $\alpha=10 \mathrm{rad} / \mathrm{s}^{2}$ and (b) for $\alpha=20 \mathrm{rad} / \mathrm{s}^{2}$.

\section{Conclusion}

The post-resonance backward whirl (Po-BW) phenomena which has been recently found to occur in rotor systems, under certain conditions, is investigated in this study with an overhung rotor system in which a snubbing contact is incorporated. The Po-BW is excited immediately after the passage through the critical FW resonance frequency during run up and coast down operations of rotor systems. This phenomenon is compared here with the well-known pre-resonance BW (PrBW) precession that precedes the critical FW resonance frequency. The whirl 
response of the considered overhung rotor system with snubbing contact is obtained by numerical simulation of the nonlinear equations of motion of the system. For all considered cases of active snubbing contact with isotropic or anisotropic stiffness in the support, the whirl response and the full spectrum analysis (FSA) have confirmed the excitation of Po-BW zones of rotational speeds. The broadness and recurrence of these zones of Po-BW rotational speeds of the system with active snubbing contact are found to be highly sensitive the snubbing stiffness level and the angular acceleration rate. The wavelet transform spectrum analysis has shown that both Pr-BW and the Po-BW possess a similar BW frequency content even though they are excited at different shaft rotational speeds. The outcomes of this study are important for pursuing further analytical, numerical and experimental verifications of Po-BW phenomena in different kinds of rotor systems working in a variety of operational conditions and subjected to different kinds of faults.

\section{Appendix}

The kinetic and potential energy equations of the considered system are written as

$$
T=\frac{1}{2} I_{0}\left(\dot{\theta}^{2}+\dot{\psi}^{2}\right)-\frac{1}{2} I_{p} \alpha t(\dot{\psi} \theta-\dot{\theta} \psi) \text { and } V=\frac{1}{2} k_{\theta} \theta^{2}+\frac{1}{2} k_{\psi} \psi^{2}
$$

The application of the Euler-Lagrange equation yields

$$
\begin{aligned}
& I_{0} \ddot{\theta}+I_{p} \alpha t \dot{\psi}+\left(k_{\theta}+\frac{1}{2} \alpha I_{p}\right) \theta=\sum M_{\theta} \\
& I_{0} \ddot{\psi}-I_{p} \alpha t \dot{\theta}+\left(k_{\psi}-\frac{1}{2} \alpha I_{p}\right) \psi=\sum M_{\psi}
\end{aligned}
$$

For the considered physical parameters of $k_{\theta}=k_{\psi}=10^{6} \mathrm{~N} / \mathrm{rad}$ and $I_{p}=0.3105 \mathrm{~kg} \cdot \mathrm{m}^{2}$ then $0.5 \alpha I_{p}=0.1553 \alpha$. Therefore, the effects of the added terms 
given by $0.5 \alpha I_{p}$ to the stiffness components in the above equations was found to be negligible for the considered acceleration rates in this study. Considering the damping, gravity, mass unbalance, snubbing and friction moments, the equations of motion can be rewritten as in Eq. (2) in the paper

$$
\begin{aligned}
& I_{0} \ddot{\theta}+I_{p} \alpha t \dot{\psi}+k_{\theta} \theta+C_{\theta} \dot{\theta}=m g a-m e a\left((\alpha t)^{2} \sin \left(0.5 \alpha t^{2}\right)-\alpha \cos \left(0.5 \alpha t^{2}\right)\right)+M_{\theta} \\
& I_{0} \ddot{\psi}-I_{p} \alpha t \dot{\theta}+k_{\psi} \psi+C_{\psi} \dot{\psi}=m e a\left((\alpha t)^{2} \cos \left(0.5 \alpha t^{2}\right)+\alpha \sin \left(0.5 \alpha t^{2}\right)\right)+M_{\psi}
\end{aligned}
$$

\section{References}

[1] Muszynska, A., Bently, D. E., Franklin, W. D., Hayashida, R. D., Kinglsey, L. M., Curry, A. E.: Influence of rubbing on rotor dynamics. In Final Report, NASA Contract No. NAS836719 https://ntrs.nasa.gov/archive/nasa/casi.ntrs.nasa.gov/19890016092.pdf (1989). Accessed May 252020

[2] Goldman, P., Muszynska, A.: Rotor-to-stator, rub-related, thermal/mechanical effects in rotating machinery. Chaos, Solitons and Fractals 5(9), 1579-1601 (1995). https://doi.org/10.1016/0960-0779(94)00165-M

[3] Jiang, J., Ulbrich, H.: Dynamics and stability of rotor/stator systems with rubs. Proceedings of the ASME Turbo Expo 4, 1-7 (2000). https://doi.org/10.1115/2000-GT0390

[4] Alber, O., Markert, R.: Rotor-Stator Contact - Overview of Current Research. MATEC Web of Conferences 16, 03001 (2014). https://doi.org/10.1051/matecconf/20141603001

[5] Howitt, F.: The Influence of Angular Acceleration Through Critical Speeds on Amplitudes of Whirl of a Rotating Shaft. McGill University http://digitool.library.mcgill.ca/thesisfile113412.pdf (1961). Accessed May 252020

[6] Zhou, S., Shi, J.: The Analytical Imbalance Response of Jeffcott Rotor During Acceleration. Journal of Manufacturing Science and Engineering 123(2), 299 (2001). https://doi.org/10.1115/1.1352021

[7] Zhou, S., Shi, J.: Optimal one-plane active balancing of a rigid rotor during acceleration. Journal of Sound and Vibration 249(1), 196-205 (2002). https://doi.org/10.1006/jsvi.2001.3660

[8] Henson, G. M.: Response of an oscillating system to harmonic forces of time-varying frequency. AIAA Journal 46(8), 2033-2041 (2008). https://doi.org/10.2514/1.34662

[9] Fleming, D. P., Sawicki, J. T., Poplawski, J. V.: Unbalance response prediction for accelerating rotors with load-dependent nonlinear bearing stiffness. ISCORMA-3 https://ntrs.nasa.gov/archive/nasa/casi.ntrs.nasa.gov/20050196666.pdf (2005). Accessed May 252020 
[10] Millsaps, K. T., Reed, G. L.: Reducing lateral vibrations of a rotor passing through critical speeds by acceleration scheduling. Journal of Engineering for Gas Turbines and Power 120(3), 615-620 (1998). https://doi.org/10.1115/1.2818190

[11] Bartha, A. R.: Dry friction backward whirl of rotor. ETH Zurich https://www.researchcollection.ethz.ch/handle/20.500.11850/145085 (2000). Accessed May 252020

[12] Qin, W., Chen, G., Meng, G.: Nonlinear responses of a rub-impact overhung rotor. Chaos, Solitons and Fractals 19(5), 1161-1172 (2004). https://doi.org/10.1016/S09600779(03)00306-0

[13] Sawicki, J. T., Padovan, J., Al-Khatib, R.: The dynamics of rotor with rubbing. International Journal of Rotating Machinery 5(4), 295-304 (1999). https://doi.org/10.1155/S1023621X99000263

[14] Azeez, M. F. A., Vakakis, A. F.: Numerical and experimental analysis of a continuous overhung rotor undergoing vibro-impacts. International Journal of Non-Linear Mechanics, 34(3) 415-435 (1998). https://doi.org/10.1016/s0020-7462(98)00022-5

[15] Ma, H., Wang, X., Niu, H., Wen, B.: Oil-film instability simulation in an overhung rotor system with flexible coupling misalignment. Archive of Applied Mechanics 85(7), 893907 (2015). https://doi.org/10.1007/s00419-015-0998-3

[16] Fan, C.-C., Syu, J.-W., Pan, M.-C., Tsao, W.-C.: Study of start-up vibration response for oil whirl, oil whip and dry whip. Mechanical Systems and Signal Processing 25(8), 31023115 (2011). https://doi.org/10.1016/j.ymssp.2011.04.012

[17] Fu, C., Ren, X., Yang, Y., Qin, W.: Dynamic response analysis of an overhung rotor with interval uncertainties. Nonlinear Dynamics 89(3), 2115-2124 (2017). https://doi.org/10.1007/s11071-017-3573-3

[18] Fu, C., Ren, X., Yang, Y., Xia, Y., Deng, W.: An interval precise integration method for transient unbalance response analysis of rotor system with uncertainty. Mechanical Systems and Signal Processing 107, 137-148 (2018). https://doi.org/10.1016/j.ymssp.2018.01.031

[19] Fu, C., Zhen, D., Yang, Y., Gu, F., Ball, A.: Effects of bounded uncertainties on the dynamic characteristics of an overhung rotor system with rubbing fault. Energies 12(22) (2019). https://doi.org/10.3390/en12224365

[20] Fu, C., Xu, Y., Yang, Y., Lu, K., Gu, F., Ball, A.: Response analysis of an accelerating unbalanced rotating system with both random and interval variables. Journal of Sound and Vibration, 466, 115047 (2020). https://doi.org/10.1016/j.jsv.2019.115047

[21] Chipato, E., Shaw, A. D., Friswell, M. I.: Effect of gravity-induced asymmetry on the nonlinear vibration of an overhung rotor. Communications in Nonlinear Science and Numerical Simulation 62, 78-89 (2018). https://doi.org/10.1016/j.cnsns.2018.02.016

[22] Chipato, E., Shaw, A. D., Friswell, M. I.: Frictional effects on the nonlinear dynamics of an overhung rotor. Communications in Nonlinear Science and Numerical Simulation 78, 104875 (2019). https://doi.org/10.1016/j.cnsns.2019.104875

[23] Bisoi, A., Samantaray, A. K., Bhattacharyya, R.: Sommerfeld effect in a gyroscopic overhung rotor-disk system. Nonlinear Dynamics 88(3), 1565-1585 (2017). https://doi.org/10.1007/s11071-017-3329-0

[24] Wu, X., Naugle, C., Meagher, J.: A Full Spectrum Analysis Methodology Applied to an Anisotropic Overhung Rotor. Journal of Applied Mechanical Engineering 5(06), 1-10 (2016). https://doi.org/10.4172/2168-9873.1000232 
[25] AL-Shudeifat, M. A.: New backward whirl phenomena in intact and cracked rotor systems. Journal of Sound and Vibration 443, 124-138 (2019).

https://doi.org/10.1016/j.jsv.2018.11.038

[26] Zilli, A., Williams, R. J., Ewins, D. J.: Nonlinear dynamics of a simplified model of an overhung rotor subjected to intermittent annular rubs. Journal of Engineering for Gas Turbines and Power 137(6) (2015). https://doi.org/10.1115/1.4028844

[27] Goldman, P., Muszynska, A.: Application of full spectrum to rotating machinery diagnostics. Orbit 20(1), 17-21 (1999).

[28] Fengqi, W., Meng, G.: Compound rub malfunctions feature extraction based on fullspectrum cascade analysis and SVM. Mechanical Systems and Signal Processing 20(8), 2007-2021 (2006). https://doi.org/10.1016/j.ymssp.2005.10.004

[29] Vakakis, A. F., Gendelman, O. V., Bergman, L. A., McFarland, D. M., Kerschen, G., Lee, Y. S.: Nonlinear targeted energy transfer in mechanical and structural systems. Springer Science \& Business Media (2008). https://doi.org/10.1007/978-1-4020-9130-8

[30] Wang, S., Bi, C., Li, J., Zheng, C.: Parametric instability of anisotropic rotor-bearing systems with a transverse crack. Journal of Sound and Vibration 443, 253-269 (2019). https://doi.org/10.1016/j.jsv.2018.11.052

[31] Jung, H. C., Krumdieck, S.: Rotordynamic modelling and analysis of a radial inflow turbine rotor-bearing system. International journal of precision engineering and manufacturing 15(11), 2285-2290 (2014). https://doi.org/10.1007/s12541-014-0592-6

[32] Hsieh, S. C., Chen, J. H., Lee, A. C.: A modified transfer matrix method for the coupling lateral and torsional vibrations of symmetric rotor-bearing systems. Journal of Sound and Vibration 289(1-2), 294-333 (2006). https://doi.org/10.1016/j.jsv.2005.02.004 